\title{
Meson spectroscopy at CLAS and CLAS12: \\ the present and the future
}

Raffaella De Vita

INFN - Genova

for the CLAS Collaboration

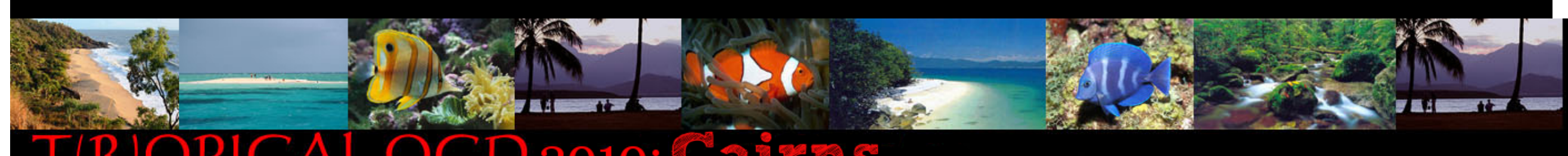




\section{Why hadron spectroscopy?}

- QCD is responsible for most of the visible mass in the universe

- Precise determination of the spectrum and properties of hadrons is necessary to reach a deep understanding of QCD

- Revealing the nature of the mass of the hadrons

- Identify the relevant degrees of freedom

- Understand the origin of confinement

- ...

- Meson spectroscopy is a key tool to investigate these issues

$<<0.1 \mathrm{fm}$

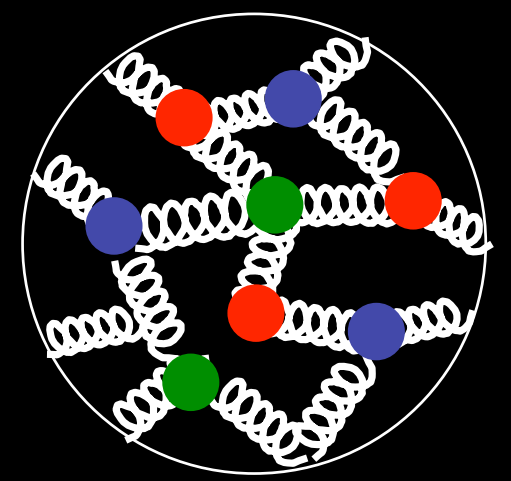

Quarks and Gluons
$0.1-1 \mathrm{fm}$

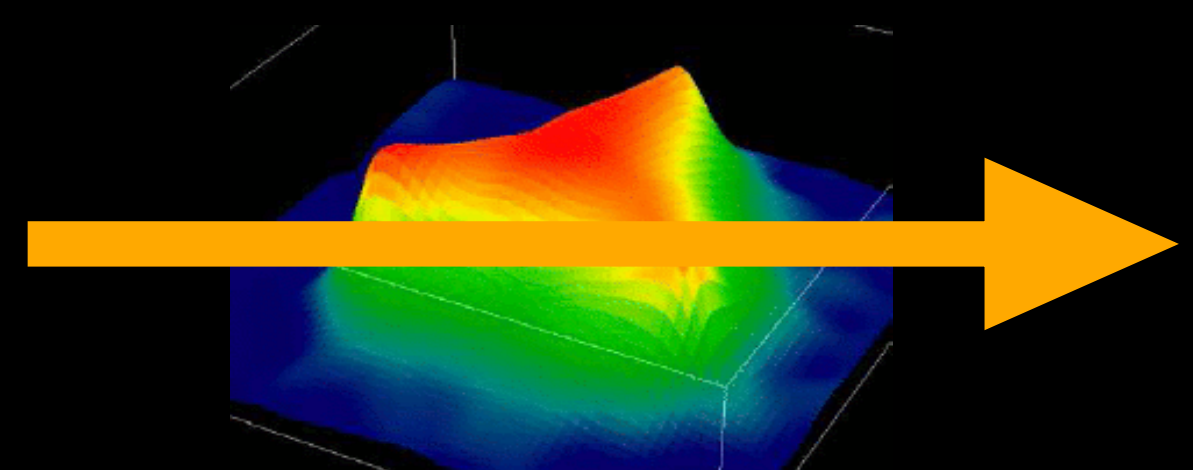

Effective Degrees of Freedom
$>1 \mathrm{fm}$

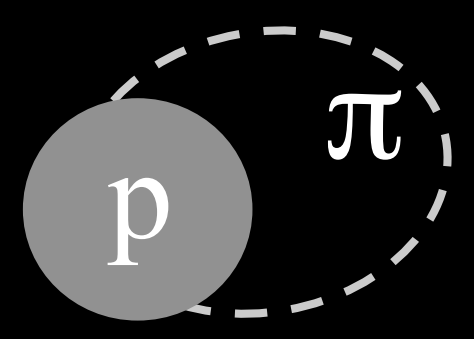

Mesons \& Baryons 


\section{Meson Spectroscopy}

\section{Objective:}

Mesons are the simplest quark bound state, i.e. the best benchmark to understand how quarks interact to form hadrons and what the role of gluons is

- precise determination of the meson spectrum

- search for unusual states as hybrids (qqg), tetraquarks (qqqq) and glueballs

\section{Technique:}

Use tagged photon beams to produce the mesonic state and isolate the single states by detecting the decay products

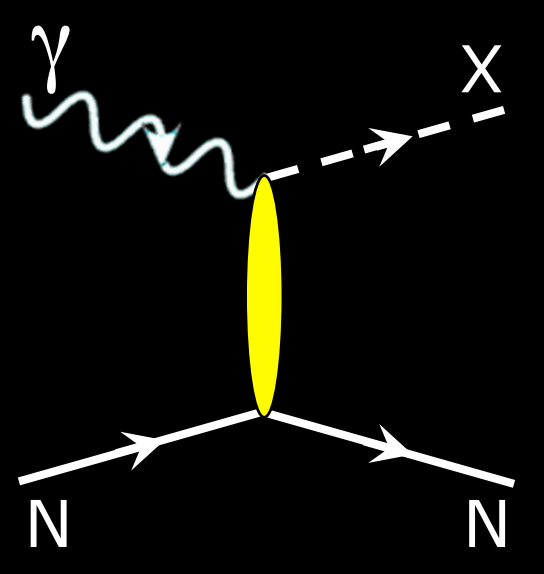

- Use of $S=1$ probe provides complementary information to $\mathrm{S}=0$ (pion beams) probes

- Measurement of the decay products and PWA to isolate single resonances

- Full determination of initial state allows to study the production mechanism

- High intensity photon beams and large acceptance detector are needed!! 


\section{Hybrids and Exotics}

A possibility to identify unambiguously a meson as an hybrid state is to look for exotic quantum numbers

* Normal mesons ( $q \bar{q})$ are classified according to their JPC where

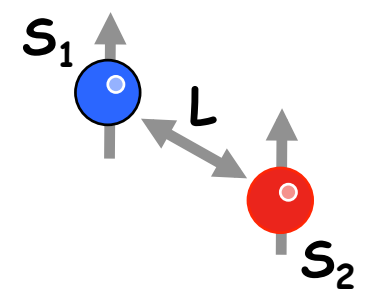

$$
\begin{aligned}
& P=(-1)^{L+1} \\
& C=(-1)^{L+S}
\end{aligned}
$$

$$
\begin{aligned}
\mathrm{JPC}^{\mathrm{PC}}= & 0^{-+} \quad \Rightarrow\left(\Pi, \mathrm{K}, \mathrm{\eta}, \mathrm{\eta}^{\prime}\right) \\
& 1^{--} \quad \Rightarrow\left(\rho, \mathrm{K}^{*}, \omega, \Phi\right) \\
& 1^{+-} \Rightarrow\left(\mathrm{b}_{1}, \mathrm{~K}_{1}, \mathrm{~h}_{1}, \mathrm{~h}_{1}{ }^{\prime}\right) \\
& \cdots
\end{aligned}
$$

* Some combinations of JPC are not allowed for conventional qव systems but can exist for "unconventional" states as hybrids

\section{Normal meson:}

flux tube in ground state

$\mathrm{m}=0, \mathrm{PC}=(-1)^{\mathrm{S}+1}$

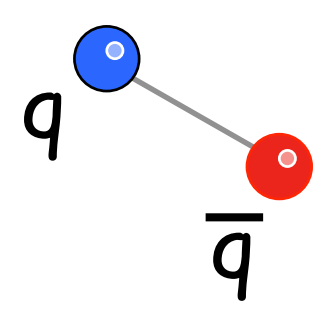

Hybrid meson:

flux tube in excited

state

$\mathrm{m}=1, \mathrm{PC}=(-1)^{\mathrm{S}}$

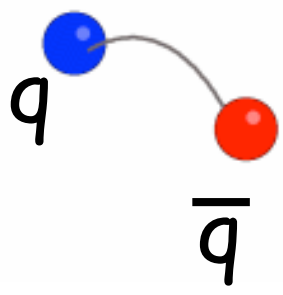

* Excitation of the flux tube leads to a new spectrum of hadrons that can have exotic quantum numbers $\quad \mathrm{JPC}^{\mathrm{PC}} \mathbf{0}^{+-}, \mathbf{1}^{-+}, \mathbf{2}^{+-} \ldots$

* Lattice QCD calculations predict masses around $2 \mathrm{GeV}$, a range that can be explored at Jefferson Lab 


\section{Search for Exotics in Photoproduction}

* Photoproduction: exotic JPC are more likely produced by $\mathrm{S}=1$ probe

Pion Beam

Quark spins

anti-aligned

$\mathrm{JPC}^{\mathrm{PC}} 1^{--}, 1^{++}$
Photon Beam

Quark spins

already aligned

$\mathrm{JC}^{\mathrm{PC}}=0^{+-}, 1^{-+}, 2^{+-}$

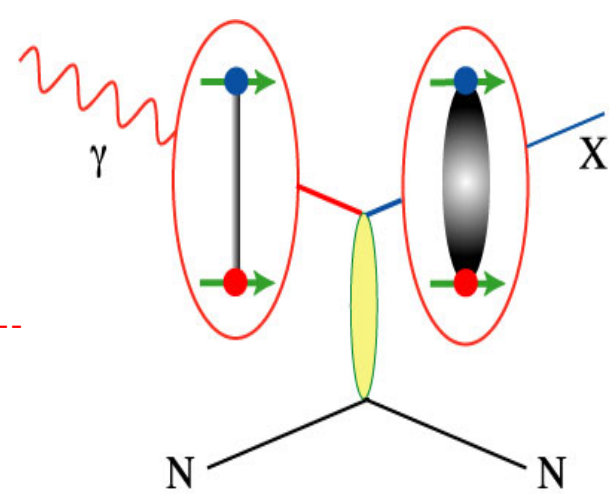

A. Szczepaniak and M. Swat, Phys. Lett. B516 (2001) 72

* Production rate for exotics is expected to be comparable to regular mesons

Few data (so far) but expected similar production rate as regular mesons

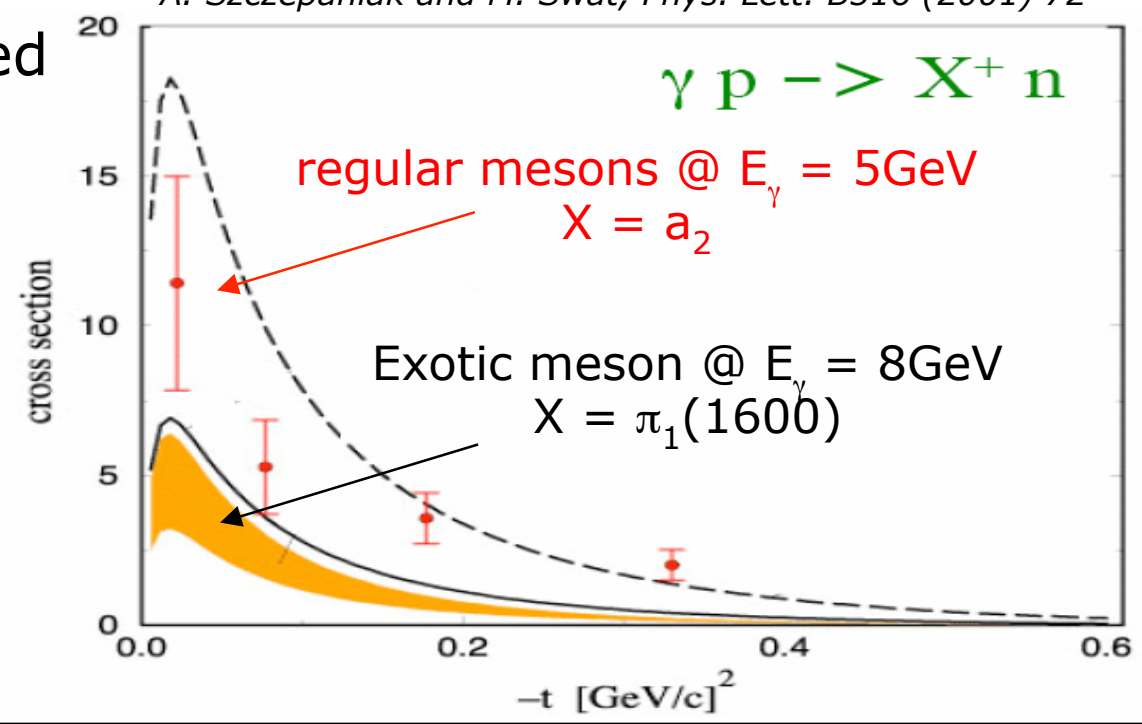




\section{Jefferson Lab}

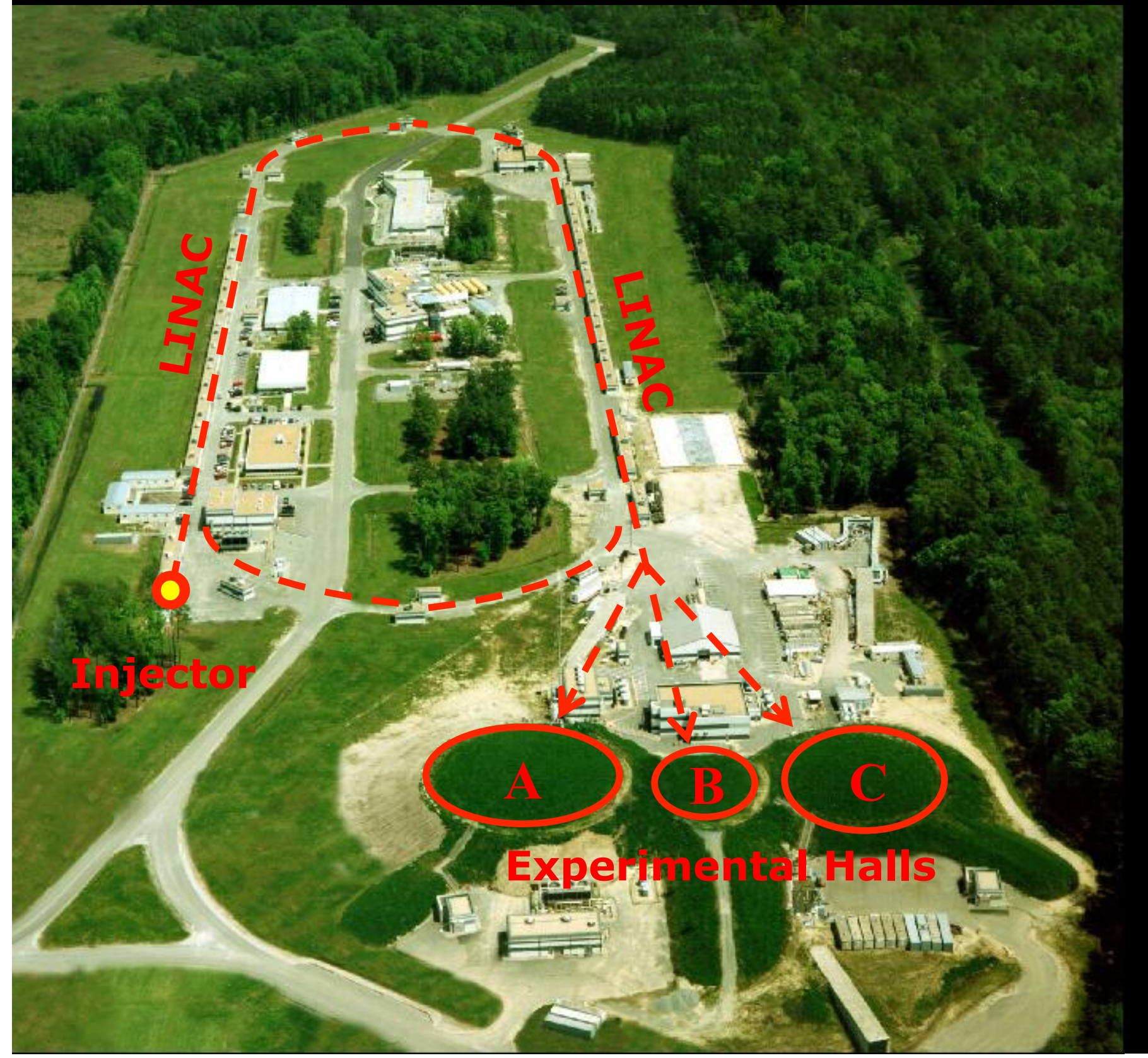

Continuous Electron

Beam

Accelerator

Facility

$\rightarrow$ E: $0.75-6 \mathrm{GeV}$

$\rightarrow \mathrm{I}_{\max }: 200 \mu \mathrm{A}$

$\rightarrow$ RF: $1499 \mathrm{MHz}$

$\rightarrow$ Duty Cycle: $~ 100 \%$

$\rightarrow \sigma(E) / E: 2.5 \times 10^{-5}$

$\rightarrow$ Polarization: $80 \%$

$\rightarrow$ Simultaneous distribution to 3 experimental Halls 


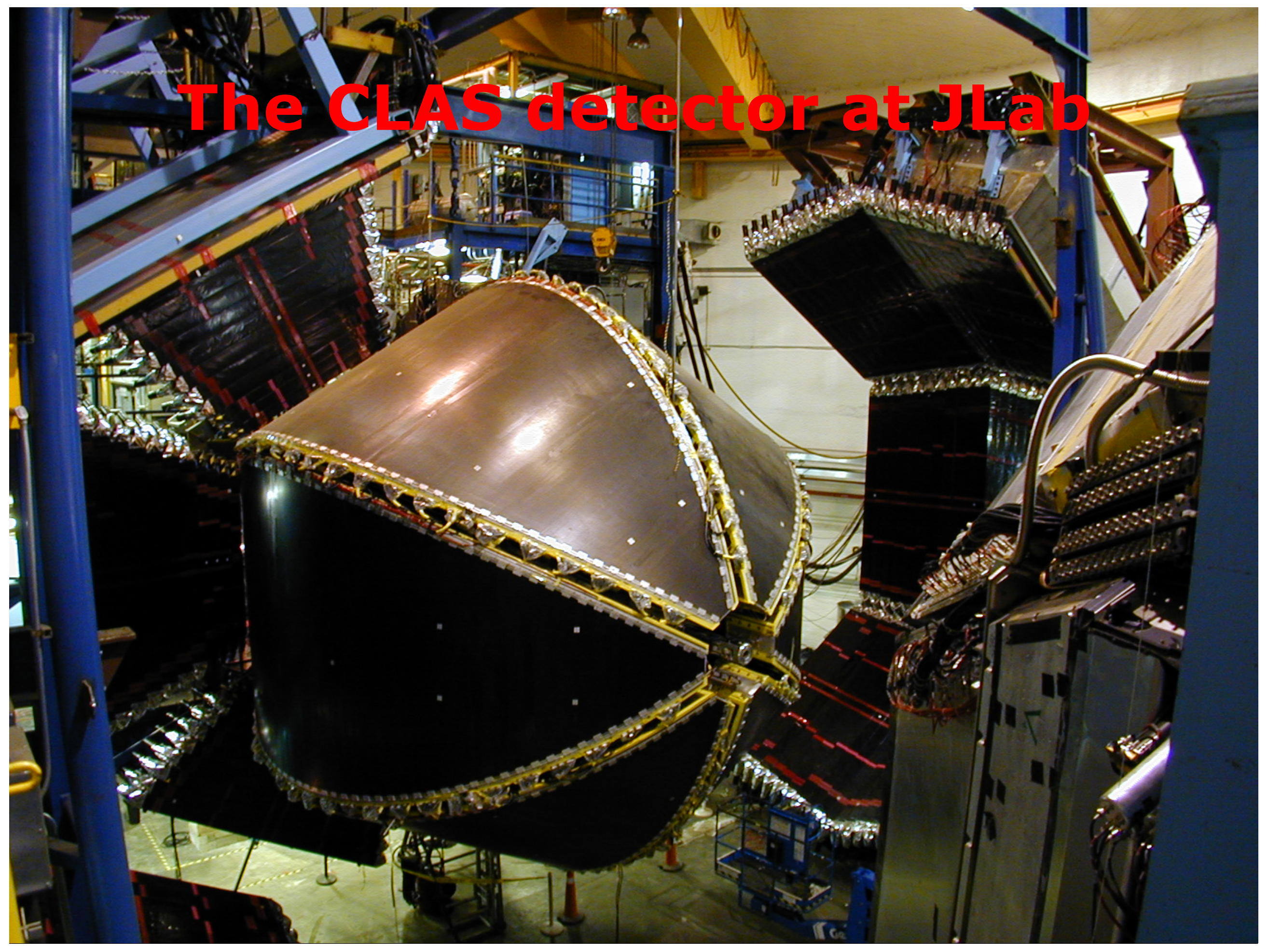




\section{CEBAF Large Acceptance Spectrometer}

\section{Torus Magnet}

$\underbrace{2}$

Target + start counter e mini-torus

Jefferson Lab

CLAS Detector

$\mathrm{O}^{\mathrm{a}} \mathrm{C}$

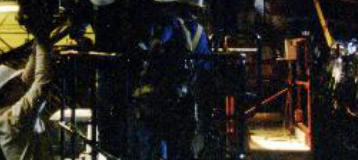

eve
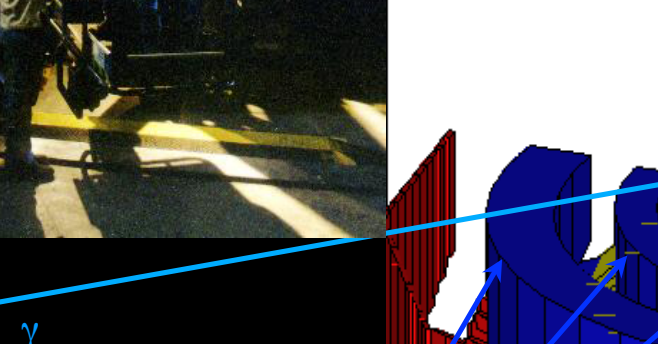

6 Superconductive Coils

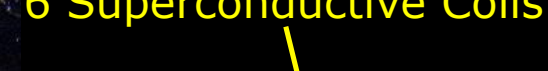

Electromagnetic Calorimeter

lead/plastic scintillator, 1296 PMTs
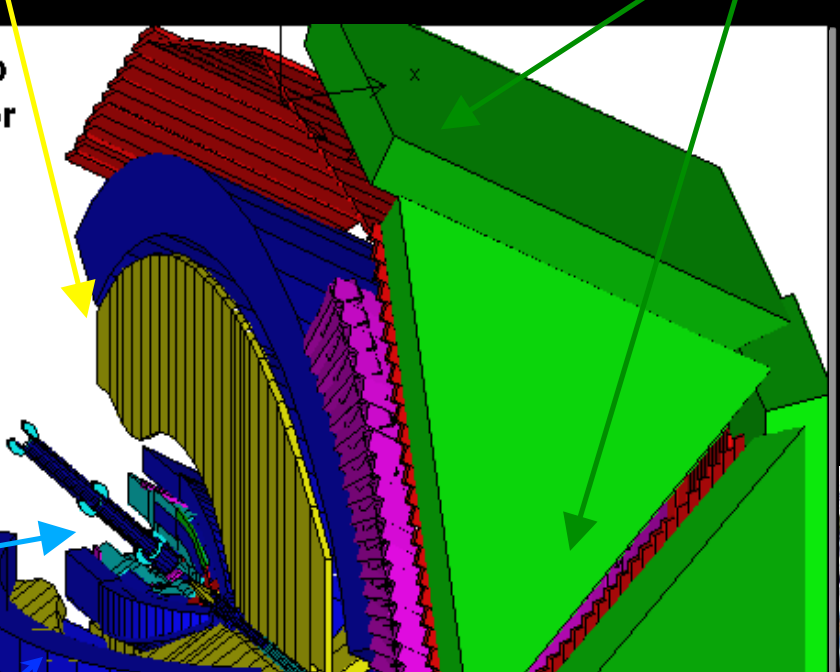

1.0

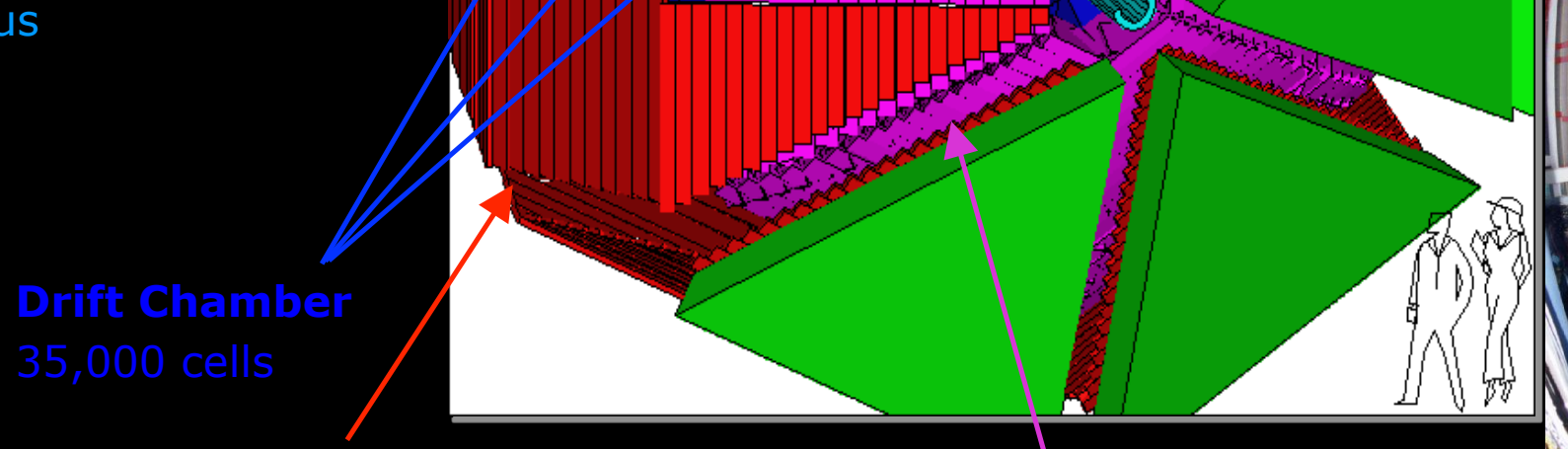

Time of Flight

Plastic Scintillator,

Cherenkov Counter

684 PMTSS Spectroscopy at CLAS and CLAS12: $\pi$ the present and the future PMTS

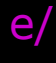




\section{Hall-B Photon Tagger}

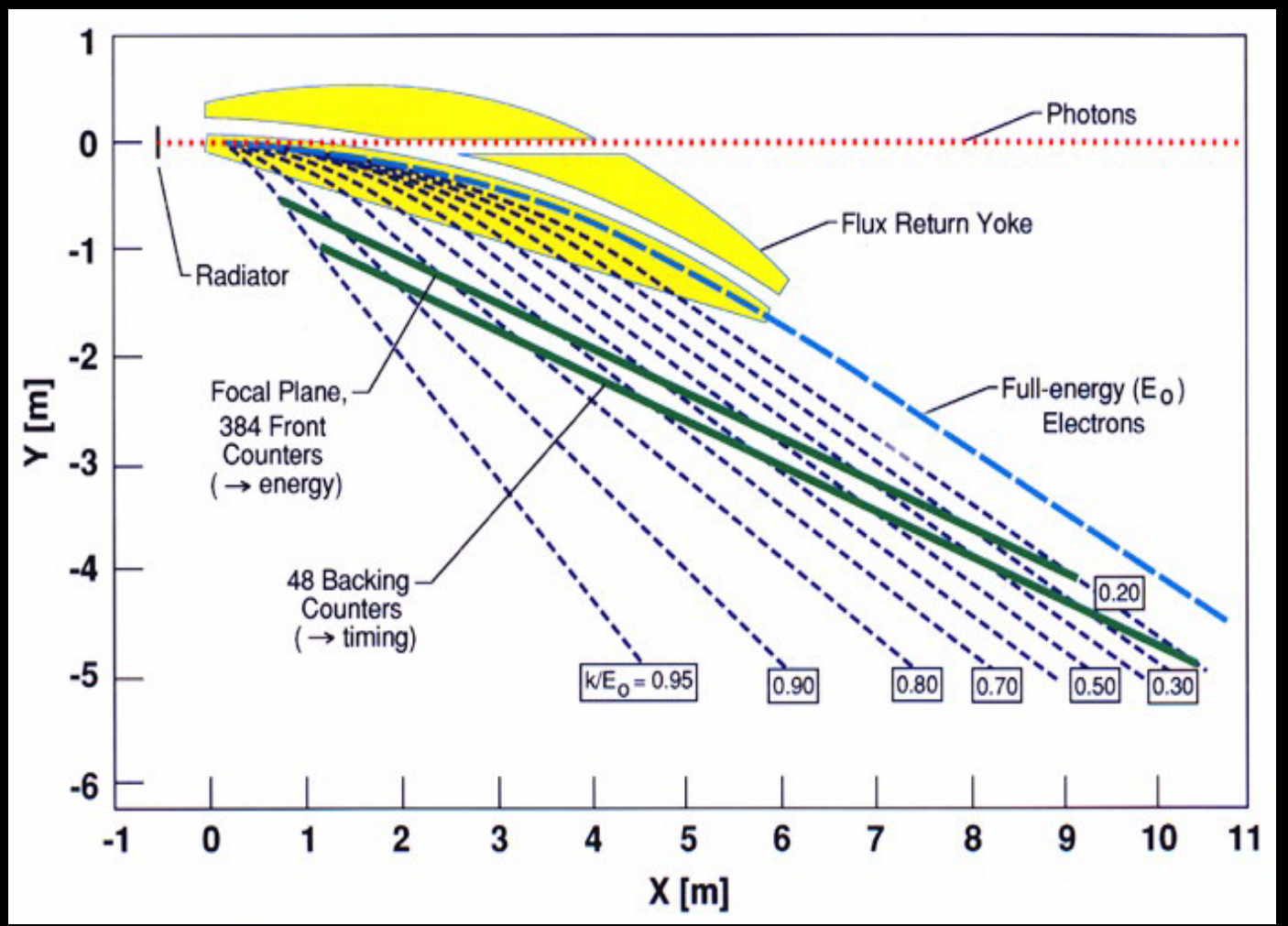

- Photon beam produced from the primary electron beam via Bremsstrahlung

- Gold and diamond radiator for In/Coherent Bremsstrahlung

- Energy coverage: 0.2-0.95 $\mathrm{E}_{0}$

- Efficiency 80\%

- Energy Resolution 10-3

- Timing Resolution 100 ps

* Maximum photon energy of $5.7 \mathrm{GeV}$

$-\mathrm{W}_{\max } \sim 3.4 \mathrm{GeV}$

- $\mathrm{M}_{\max } \sim 2.5 \mathrm{GeV}$

* Beam intensity $10^{7} \mathrm{\gamma} / \mathrm{s}$

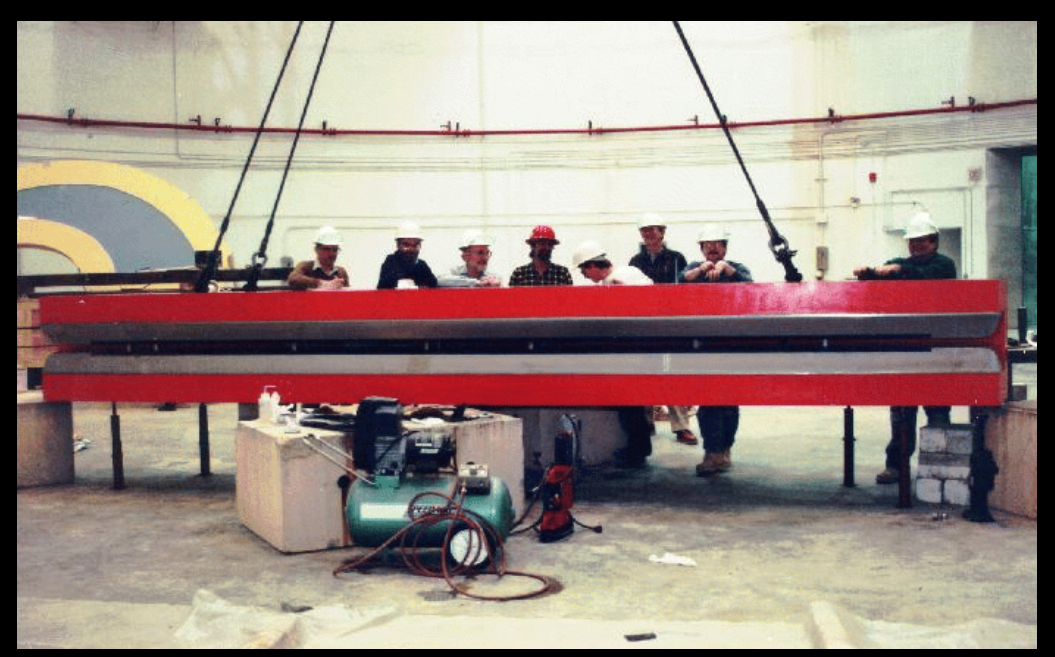




\section{Partial Wave Analysis}

\section{1) Isobar Model}

e.g. $3 \pi$

system

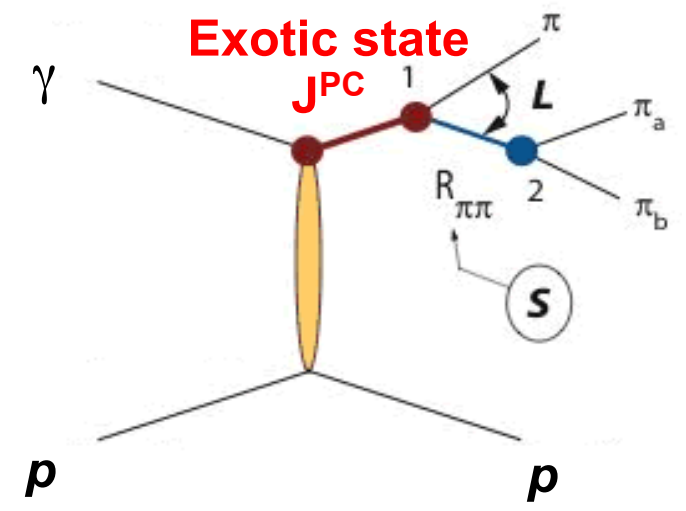

(a) resonance: $X$ decay

$X\left(2^{-+}\right) \rightarrow f_{2}(1275) \pi$

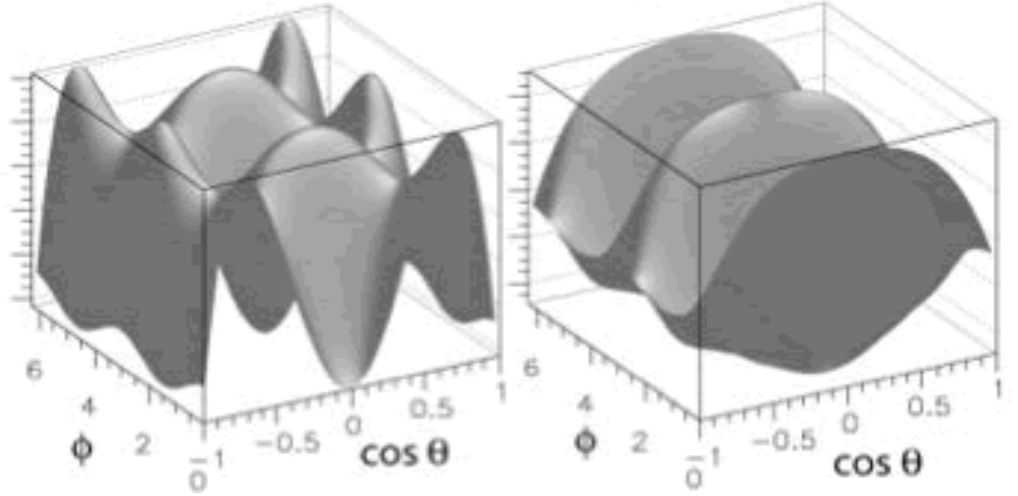

2) Moments+Dispersion Relations

1) Moments of the angular distribution in term of partial waves

$$
\begin{aligned}
& \left\langle Y_{\lambda \mu}\right\rangle\left(E_{\gamma}, t, M\right)=\frac{1}{\sqrt{4 \pi}} \int d \Omega_{\pi} \frac{d \sigma}{d t d M d \Omega_{\pi}} Y_{\lambda \mu}\left(\Omega_{\pi}\right) \\
& \left\langle Y_{00}\right\rangle=N\left[|S|^{2}+\left|P_{-}\right|^{2}+\left|P_{0}\right|^{2}+\left|P_{+}\right|^{2}+\left|D_{-}\right|^{2}\right. \\
& \left.+\left|D_{0}\right|^{2}+\left|D_{+}\right|^{2}+\left|F_{-}\right|^{2}+\left|F_{0}\right|^{2}+\left|F_{+}\right|^{2}\right]
\end{aligned}
$$

2) Parametrize partial waves in term of known $\pi \pi$ phase shift and unknown coefficients using Dispersion Relations

3) Derive partial wave cross sections to compare with models

e.g. $2 \pi$ system
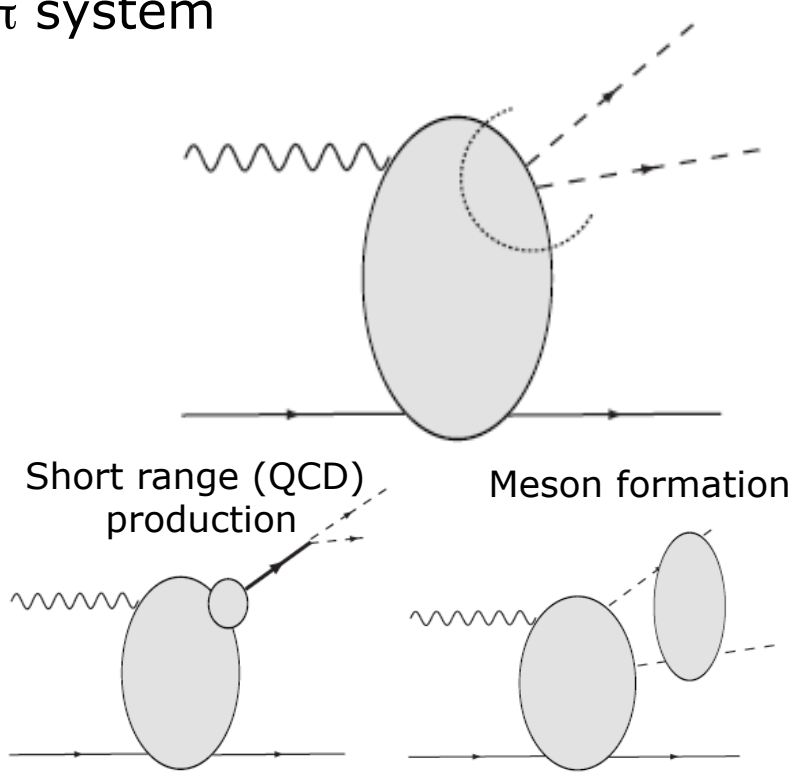


\section{PWA with Isobar Model}

$$
\gamma p \rightarrow \pi^{+} \pi^{+} \pi^{-}(n)
$$

- Possible evidence of exotic meson $\pi_{1}(1600)$ in $\pi p \rightarrow(3 \pi)^{-} p$ (E852Brookhaven)

> Not confirmed in a re-analysis of a higher statistic sample

> New evidence recently reported by Compass

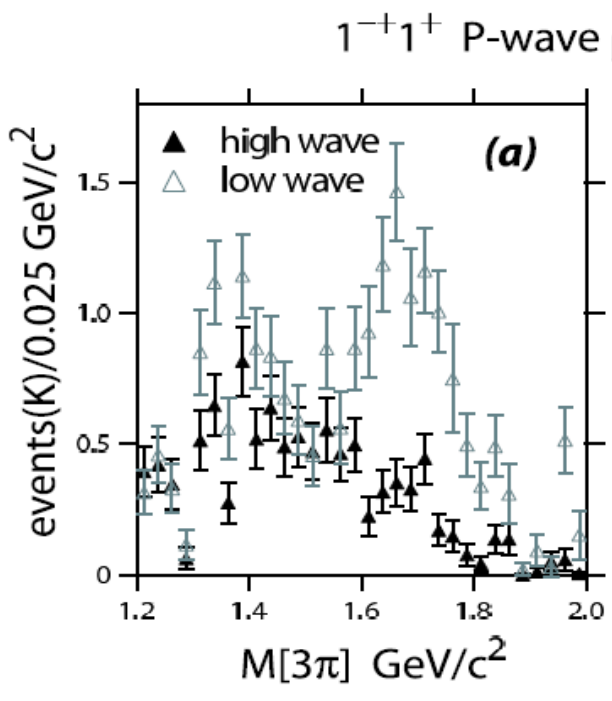

$\left(\pi^{-} \pi^{-} \pi^{+}\right)$

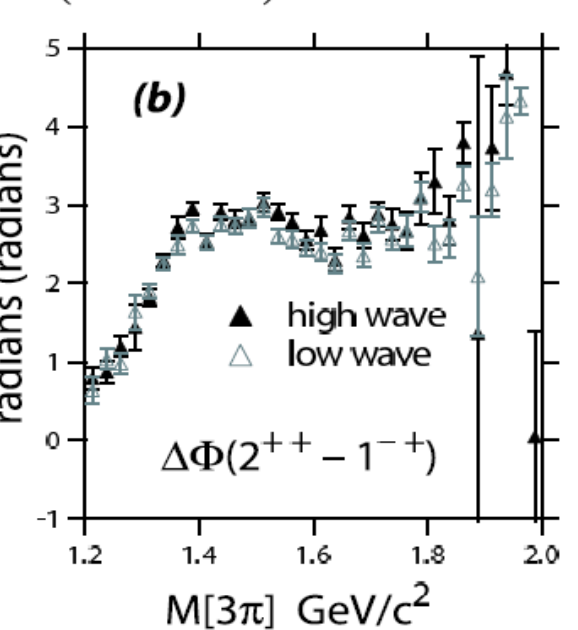

\section{CLAS-g6C}

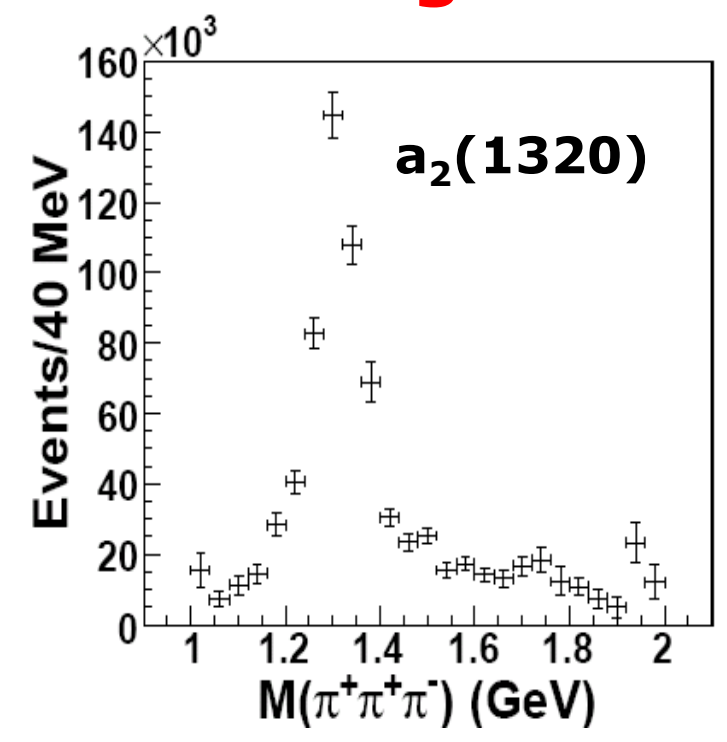

$>$ Clear evidence of non-exotic $2^{++}$state $a_{2}(1320)$

$>$ No-evidence of exotic $1^{-+}$state $\pi_{1}(1600)$

> Relevance of baryon resonance background

\section{PWA in CLAS is feasible!}




\section{PWA: Moments+Dispersion Relations}

$$
\gamma \mathrm{p} \rightarrow \mathrm{p} \pi^{+} \pi^{-}
$$

* Analysis of CLAS-g11 data

* $M\left(\pi^{+} \pi^{-}\right)$spectrum below $1.5 \mathrm{GeV}$ :

-P-wave: $\rho$ meson

-D-wave: $f_{2}(1270)$

-S-wave: $\sigma, f_{0}(980)$ and $f_{0}(1320)$

* Moments of the 2-pion angular distribution extracted via likelihood fit of data

* Partial Wave fitted to experimental moments

* Known states well reproduced, e.g. $\rho(770)$

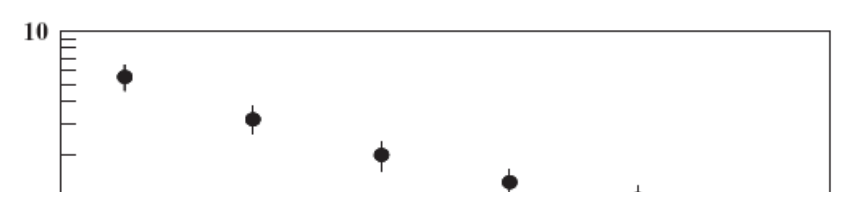

First observation of $f_{0}(980)$ in photoproduction

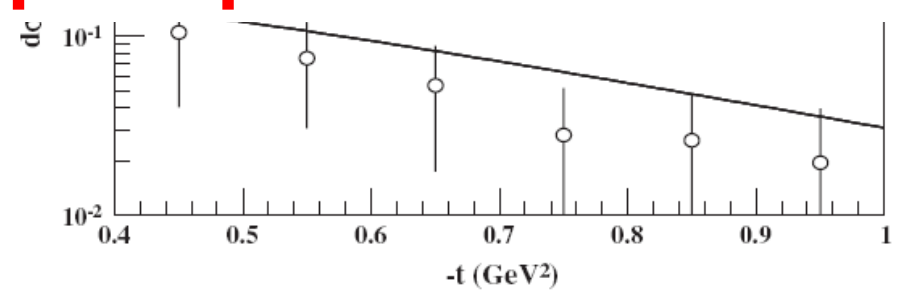

$3.4 \mathrm{GeV}<\mathrm{E}_{\mathrm{\gamma}}<3.6 \mathrm{GeV}$

$0.5 \mathrm{GeV}^{2}<-\mathrm{t}<0.6 \mathrm{GeV}^{2}$
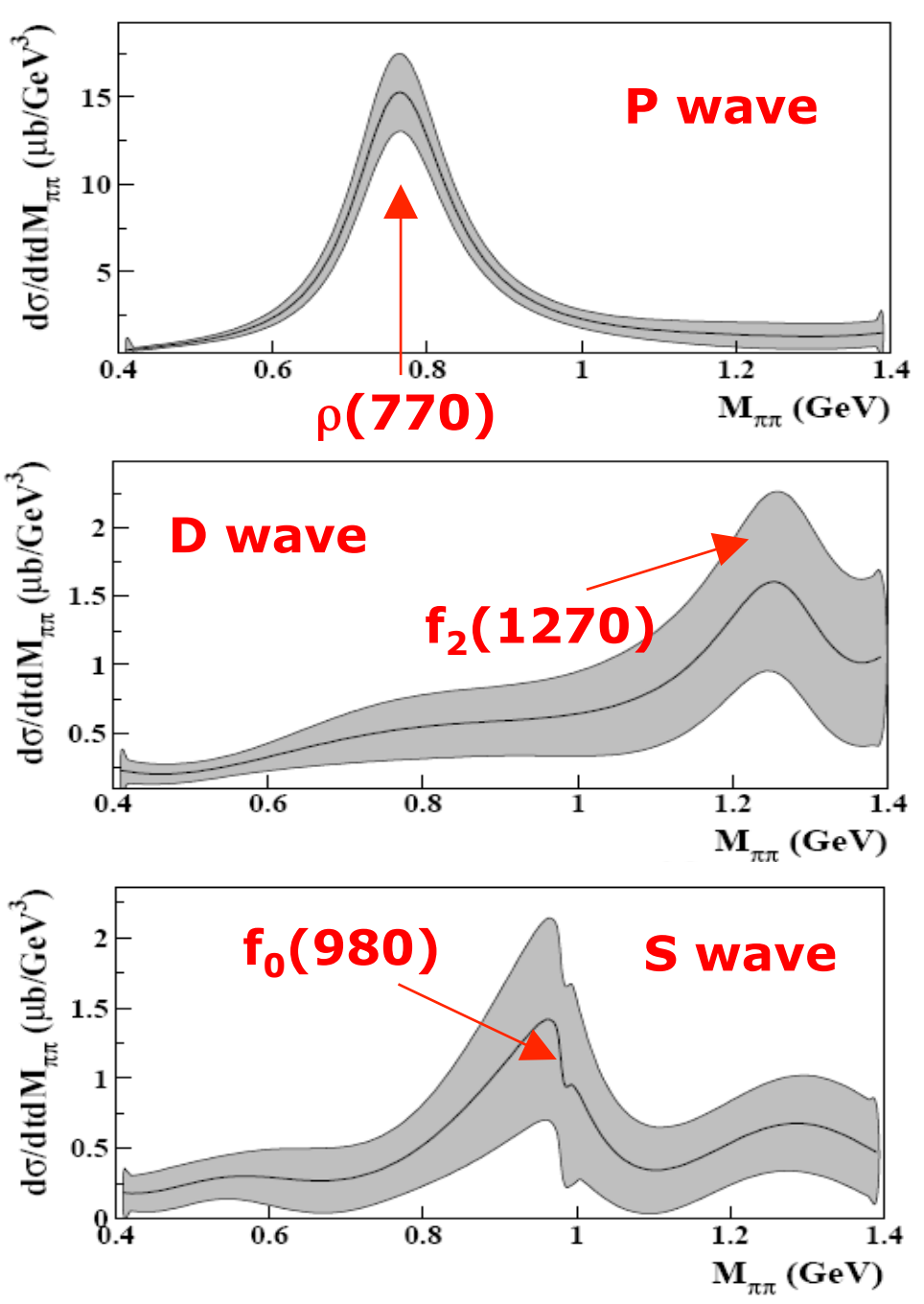


\section{New High Statistics Photon run: $g 12$}

Search for new forms of hadronic matter in photoproduction on the proton

- Data taking completed in 2008

- Photon Energy up to $5.5 \mathrm{GeV}$

- More than 26 billion triggers (2-prong + 3-prong)

- Total Luminosity: $68 \mathrm{pb}^{-1}$

- Data processing completed and physics analysis in progress

Several exclusive channels are being analyzed

$$
\begin{aligned}
& \gamma \mathrm{p} \rightarrow \pi^{+} \pi^{+} \pi^{-}(\mathrm{n}) \\
& \gamma \mathrm{p} \rightarrow \mathrm{K}^{+} \mathrm{K}^{+} \Xi^{*-}(1530) \\
& \gamma \mathrm{p} \rightarrow \mathrm{pK}^{+} \mathrm{K}^{-}(\eta / \pi) \\
& \gamma \mathrm{p} \rightarrow \mathrm{p} \pi^{+} \pi^{-}(\eta) \\
& \gamma \mathrm{p} \rightarrow \pi^{+} \mathrm{K}^{+} \mathrm{K}^{-}(\mathrm{n}) \\
& \gamma \mathrm{p} \rightarrow \mathrm{e}^{+} \mathrm{e}^{-} \mathrm{p} \\
& \ldots
\end{aligned}
$$

- Cascade spectroscopy

- Search for exotic mesons

- Study of strangeonium states

- ... 


\section{EG6: Meson spectroscopy in coherent production on ${ }^{4} \mathrm{He}$}

- search for exotics in $\pi \eta, \pi \eta$ ' final states

- $6 \mathrm{GeV}$ electron beam on taraet on hiah pressure aas taraet

- scattered electron at "0" dearees $\rightarrow$ auasi-real photo-production

- recoiling nucleus detected in Radial TPC

- hadronic final state detected in CLAS

- data taking completed in fall 2009

- analysis in progress

Analysis of existing CLAS data shows ciear peaks associated tóget knownomesensiliprward-focusing of

Moeller electrons and bending of recoepur(@):

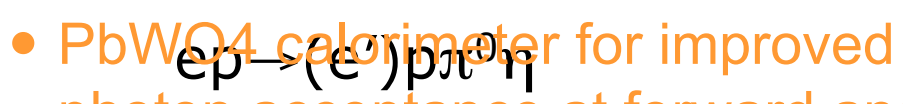
photon acceptance at forward anglessoo The Technique works!!

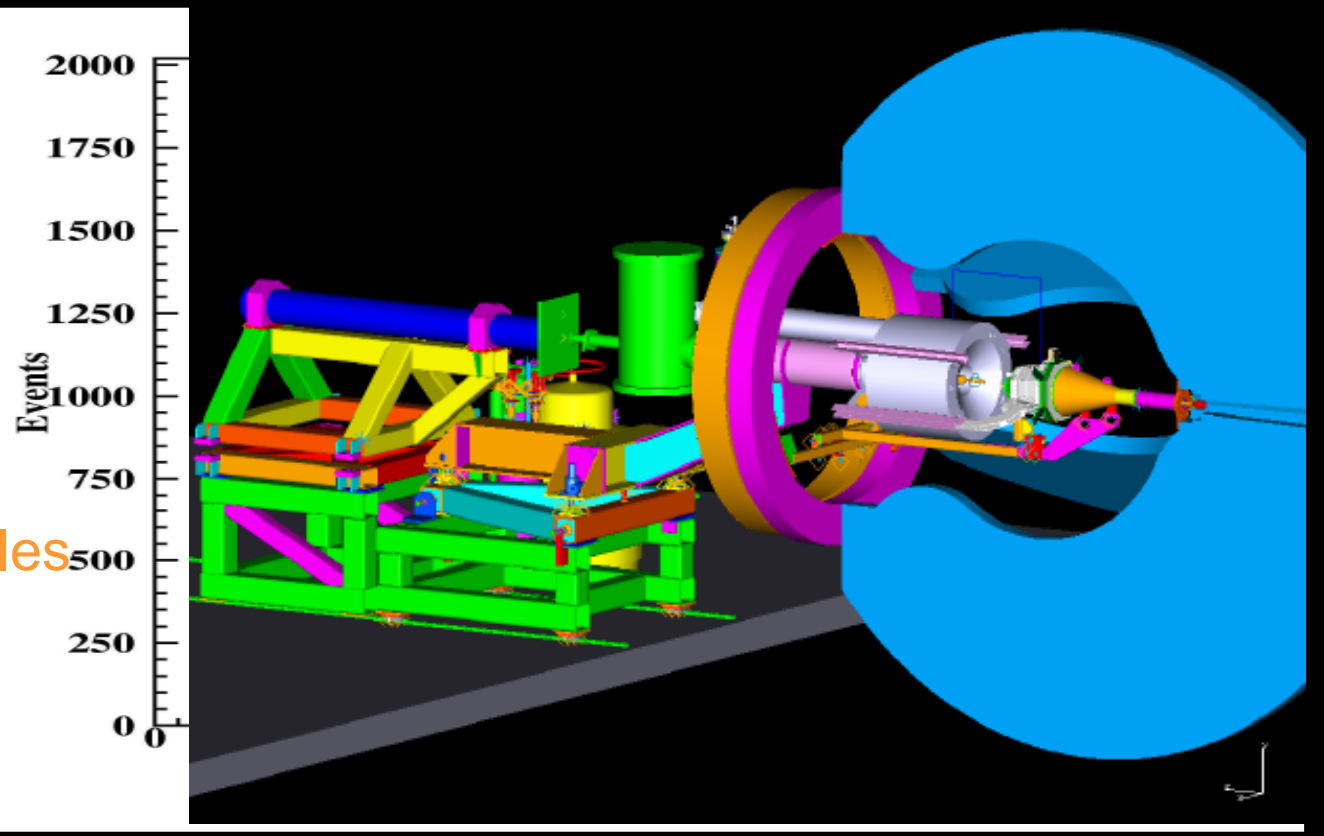




\section{$12 \mathrm{GeV}$ CEBAF}

Construction of the new Hall D

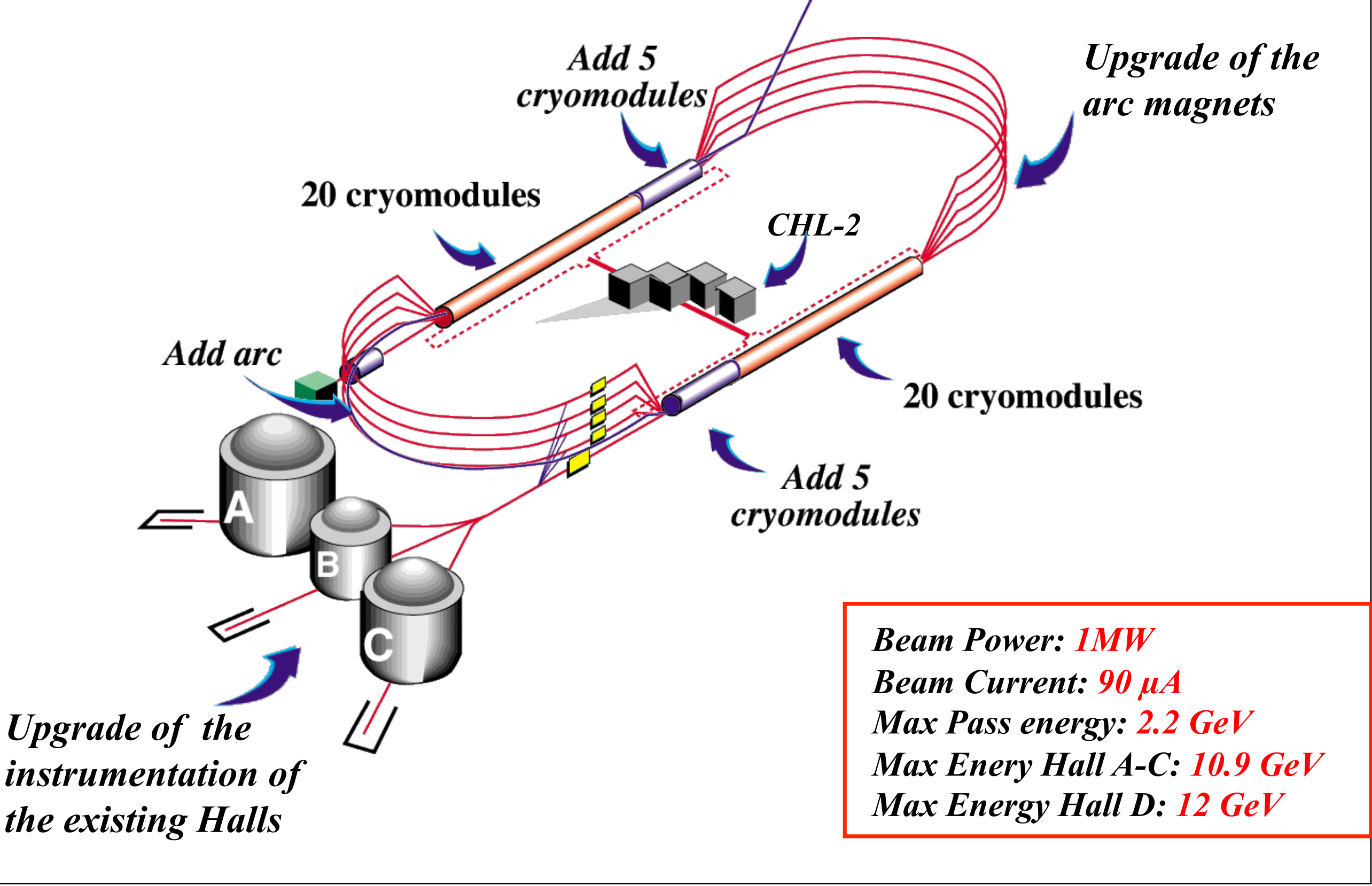




\section{CLAS12}

Forward Detector:

- TORUS magnet

- Forward SVT tracker

- HT Cherenkov Counter

- Drift chamber system

- LT Cherenkov Counter

- Forward ToF System

- Preshower calorimeter

- E.M. calorimeter

Central Detector:

- SOLENOID magnet

- Barrel Silicon Tracker

- Central Time-of-Flight

Proposed upgrades:

- Micromegas (CD)

- Neutron detector (CD)

- RICH detector (FD)

- Forward Tagger (FD)

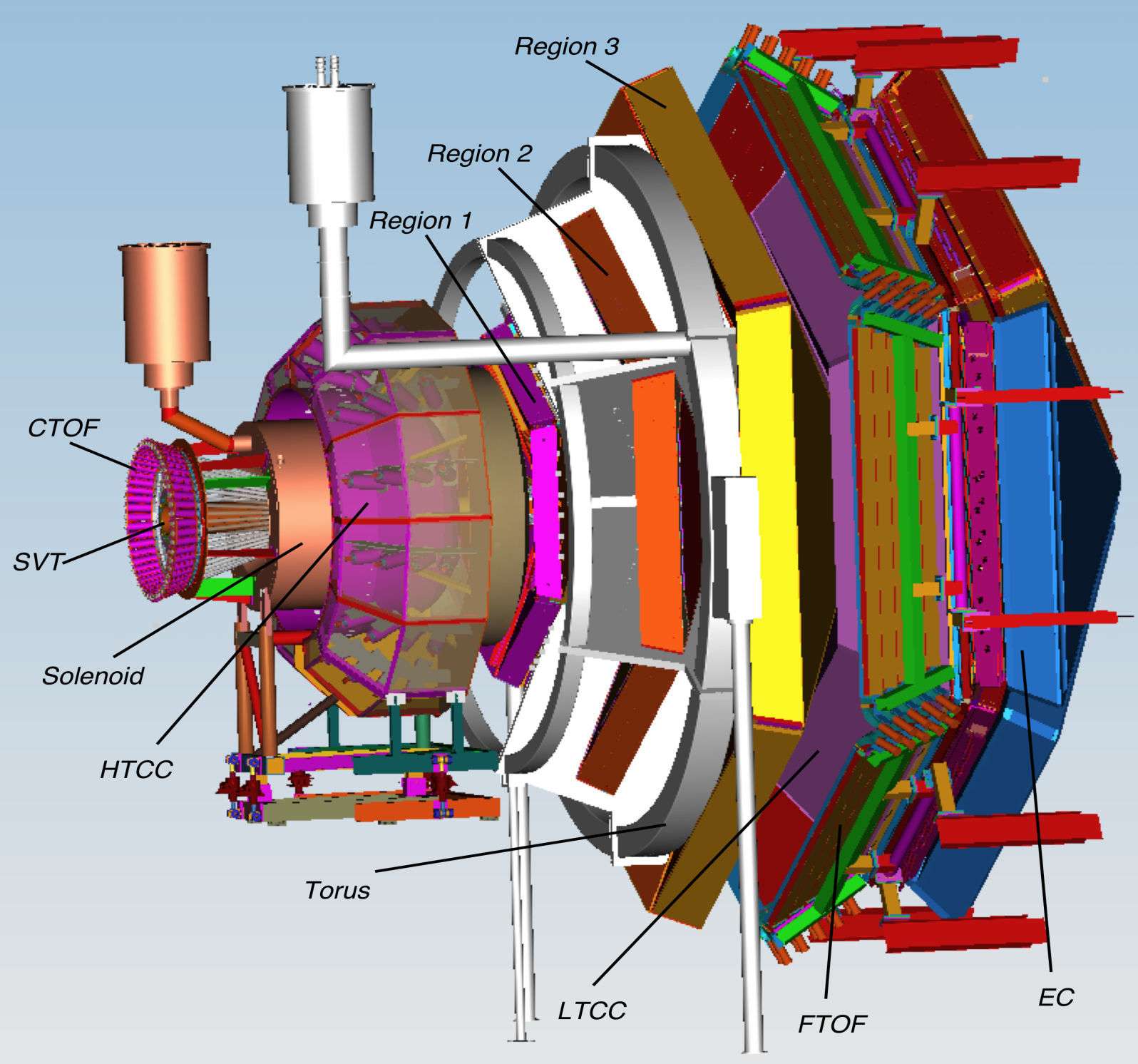




\section{Spectroscopy with CLAS12}

The construction of a Forward Tagger has been proposed to continue the spectroscopy program with CLAS12 using quasi-real photoproduction

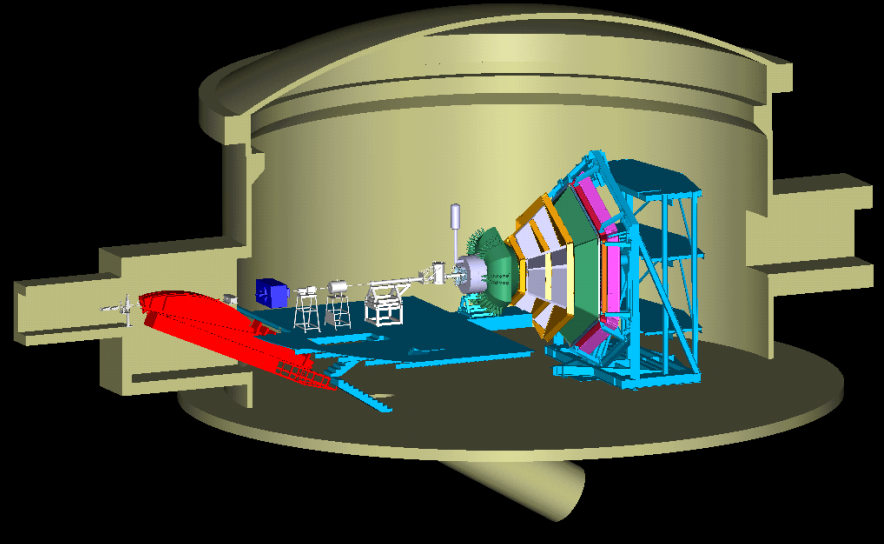

- electron scattering at "0" degrees (LowQ, posttarget tagging)

- low-Q2 virtual photon $\Leftrightarrow$ real photon (well known technique from high energy experiments)

- detection of the scattered electron allows to determine the photon energy and polarization

- knowledge of the photon energy and electron kinematics are important to select the exclusive final state

- polarization is essential to isolate the exclusive production mechanisms (M) and acts as a JPC filter if $M$ is known

- high luminosity on thin (gas)- targets

- Complementary technique to Hall-D Coherent Bremsstrahlung photon beam 


\section{A Forward Photon Tagger for CLAS12}

Quasi-real photo-production with detection of scattered electrons at " 0 " degrees

- electron beam on target, scattered electrons at small angles are detected

- hadronic final state is detected in CLAS12

- photon linear polarization 65\% - $20 \%$

- high electromagnetic background and radiation levels

- limited space

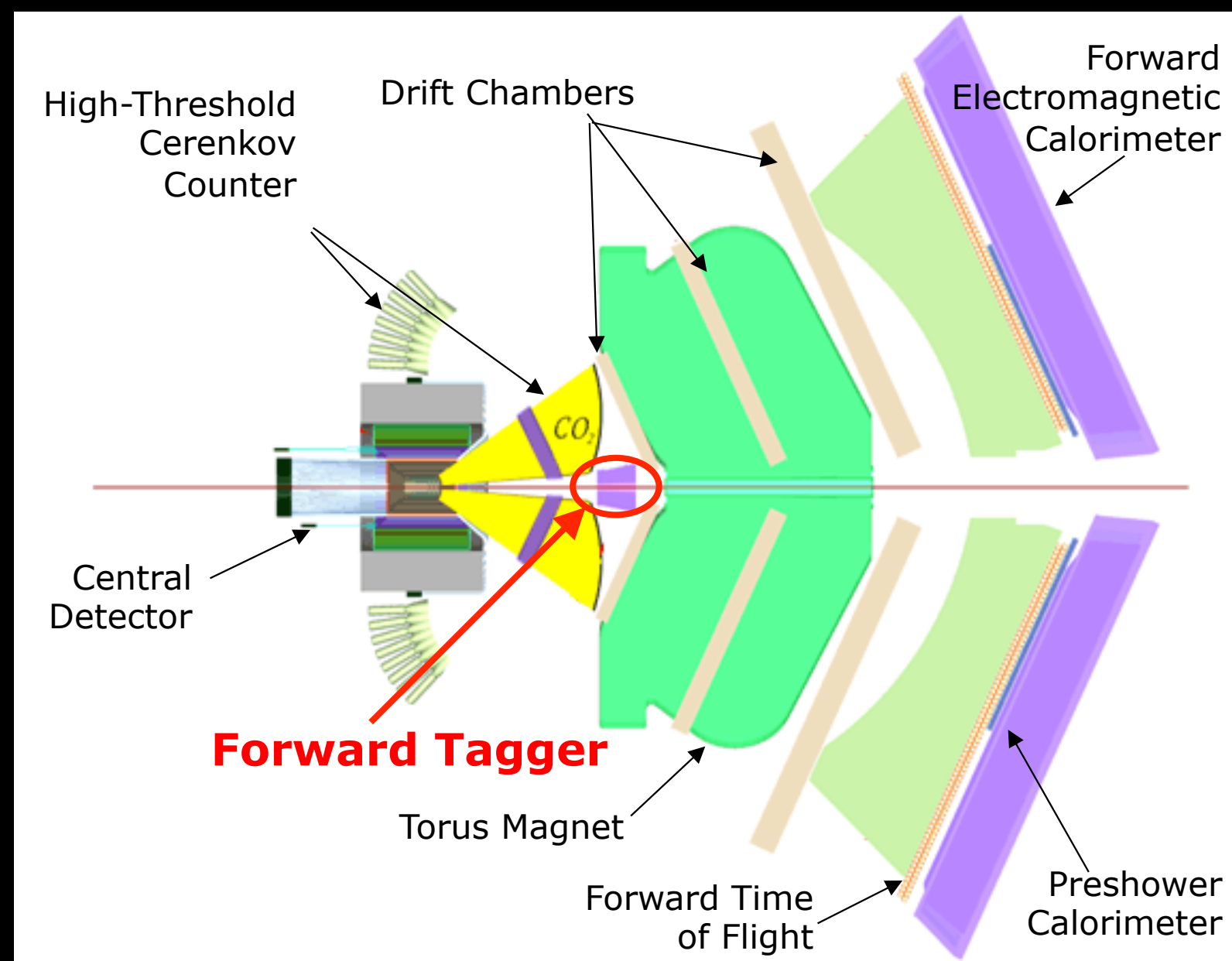




\section{Forward Tagger}

Electron detection via Calorimeter+Tracker+Veto for determination of energy and angles

- calorimeter to determine the electron energy with few \% accuracy

- tracker to determine precisely the electron scattering plane and the photon polarization

- veto to distinguish electrons from photons and for fast triggering

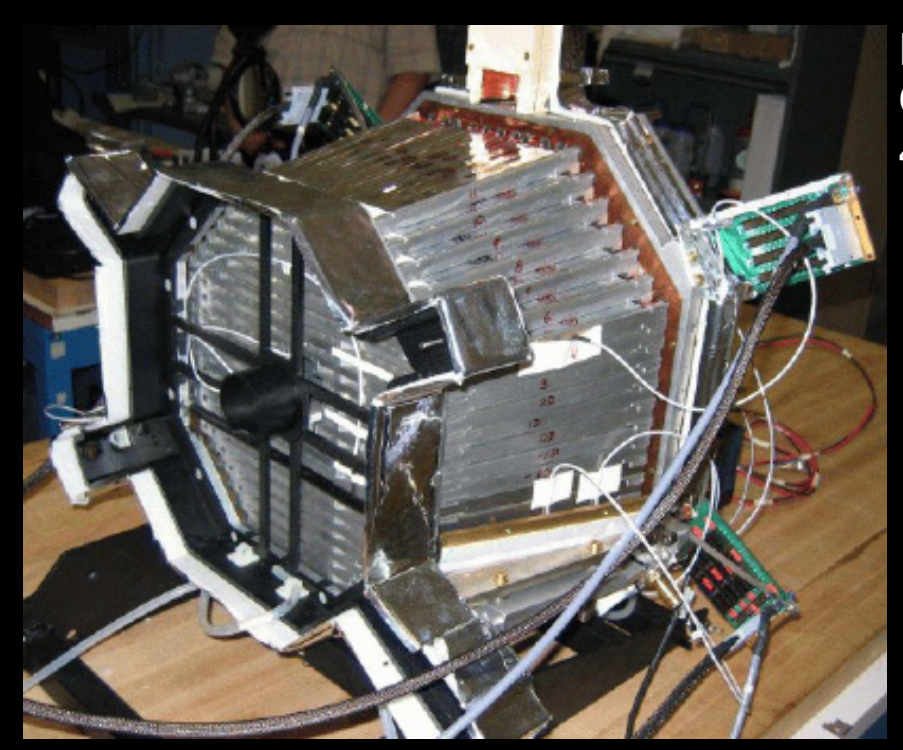

PbW04

Calorimeter 424 Crystals

\begin{tabular}{|l|l|}
\hline \multicolumn{2}{|l|}{ Electron Detector } \\
\hline $\mathrm{E}^{\prime}$ & $0.5-4 \mathrm{GeV}$ \\
\hline$v$ & $7-10.5 \mathrm{GeV}$ \\
\hline$\theta$ & $2-5 \mathrm{deg}$ \\
\hline $\mathrm{Q}^{2}$ & $0.007-0.3 \mathrm{GeV}^{2}$ \\
\hline Photon Flux & $5 \times 10^{7} \gamma / \mathrm{s} @ \mathrm{~L}_{\mathrm{e}}=10^{35}$ \\
\hline Rate & $20 \mathrm{MHz} @ \mathrm{~L}_{\mathrm{e}}=10^{35}$ \\
\hline
\end{tabular}

\section{Different hardware options:}

\section{Calorimeter:}

- homogenous crystals (PbW04, LYSO, ..)

- sampling device (W+Fibers, W+Si, ...)

Tracker:

- gems, micromegas, ...

Veto:

- Plastic scintillator tiles, sci-fibers , ... 


\section{GEANT4 Simulations}

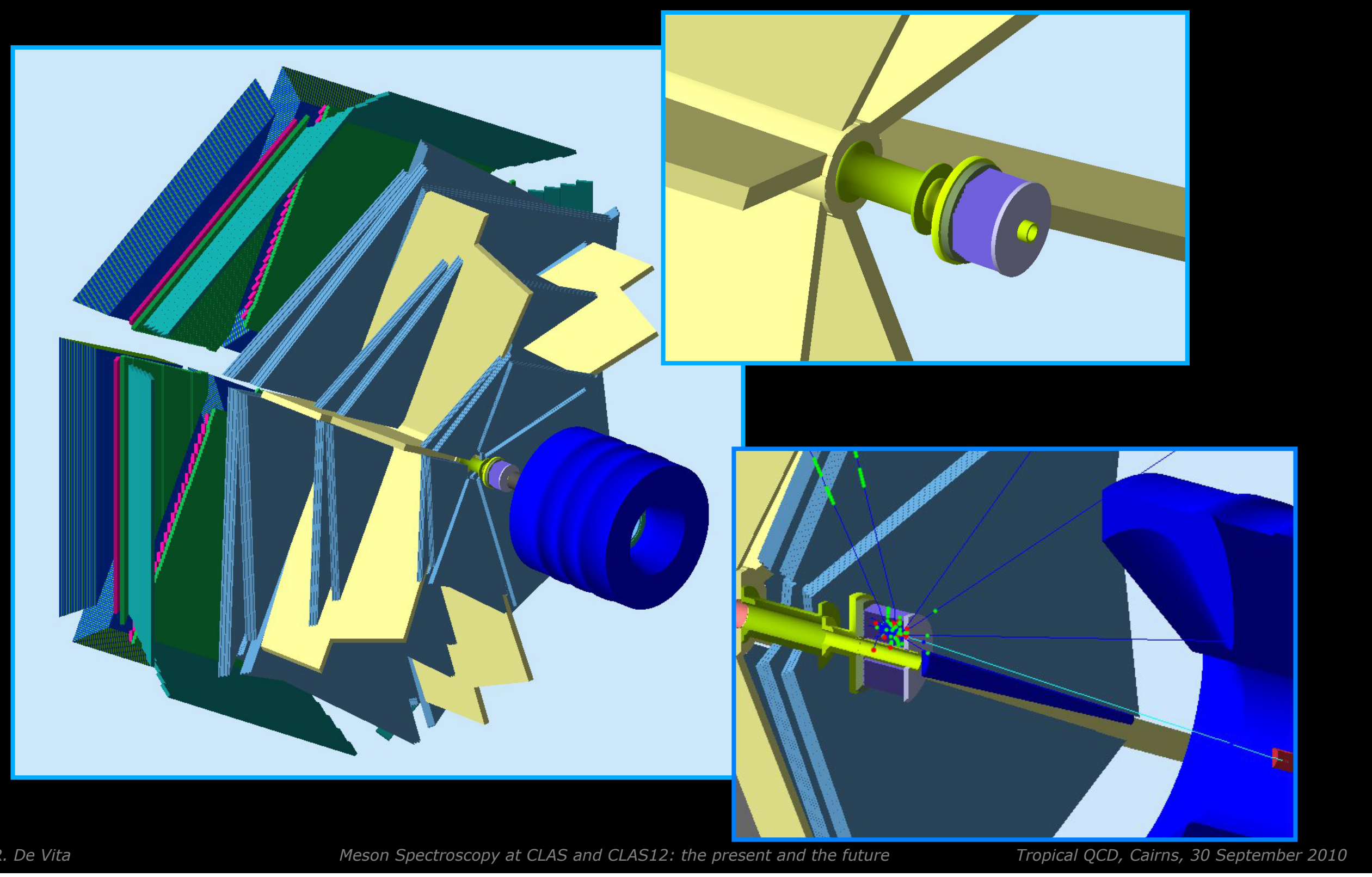




\section{A New Physics Program}

A tagged photon beam in CLAS12 will open new possibilities for high quality physics beyond the already approved program:

- Meson spectroscopy

- Spectroscopy on $\mathrm{H}$ target and search for exotics

- Spectroscopy on ${ }^{4} \mathrm{He}$ and other gas targets

- Hadron spectroscopy

- Heavy mass baryon resonances (Cascades and $\Omega^{-}$)

- Compton scattering

- Meson polarizabilities

- $\mathrm{J} / \Psi$ production close to threshold and on nuclear targets

- Large - $t$ physics

LOI-2010-001: "Hadron Spectroscopy with Low Q2 electro-scattering in CLAS"

LOI-2010-004: "Production of the Strangest Baryon with CLAS12" Endorsed by PAC35 and full proposal in preparation 


\section{Summary}

Meson spectroscopy is a fundamental tool to study the properties of strong interaction

The present at CLAS

- Meson spectrum investigated in photoproduction

- PWA (IM and Moments + Dispersion relations) feasible in CLAS

- First results published, experimental program continues...

The future at CLAS12

- Experimental program can continue using quasi-real photoproduction

- A new forward tagger is being designed to detect scattered electrons down to 2 deg.

- High intensity (quasi)-real photon beam on proton and nuclear targets will give access to a rich physics program

- LOIs presented to PAC35 have been approved

- Full proposal is being prepared for January 2011 


\section{Search for Hybrid Mesons}

Understanding the role of gluons and the origin of confinement is crucial to complete our picture of strong interaction

- At high energy experimental evidence is found in jet production

- At lower energies the hadron spectrum carries information about the gluons that bind quarks

- Can we find hints of the glue in the meson spectrum?

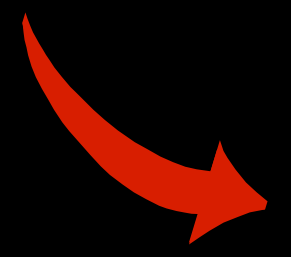

\section{Search for non-standard states with explicit gluonic degrees of freedom}

- Existence of these state is not prohibited by QCD but is not yet firmly established experimentally

- Finding clear evidence for hybrid mesons and determining their spectrum is of fundamental importance

Regular meson

q $\bar{q}$

Hybrid meson

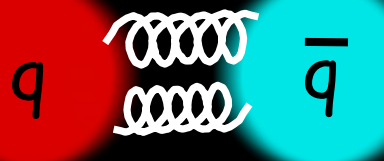




\section{CLAS12 - Design parameters}

\begin{tabular}{|c|c|c|c|}
\hline Forward Detector & & $\begin{array}{l}\text { Forward } \\
\text { Detector }\end{array}$ & $\begin{array}{l}\text { Central } \\
\text { Detector }\end{array}$ \\
\hline & $\begin{array}{c}\text { Angular range } \\
\text { Tracks } \\
\text { Photons } \\
\end{array}$ & $\begin{array}{l}5^{0}-40^{0} \\
3^{0}-40^{0}\end{array}$ & $\begin{array}{l}35^{0}-125^{0} \\
\text { n.a. }\end{array}$ \\
\hline & $\begin{array}{l}\text { Resolution } \\
\qquad \begin{array}{r}\delta / \mathrm{p}(\%) \\
\delta \theta(\mathrm{mr}) \\
\Delta \phi(\mathrm{mr})\end{array}\end{array}$ & $\begin{array}{l}<1 @ 5 \mathrm{GeV} / \mathrm{c} \\
<1 \\
<3\end{array}$ & $\begin{array}{l}<5 @ 1.5 \mathrm{GeV} / \mathrm{c} \\
<10-20 \\
<5\end{array}$ \\
\hline 5 & $\begin{array}{l}\text { Photon detection } \\
\text { Energy (MeV) } \\
\qquad \theta(\mathrm{mr})\end{array}$ & $\begin{array}{l}>150 \\
<4 @ 1 \mathrm{GeV}\end{array}$ & $\begin{array}{l}\text { n.a. } \\
\text { n.a. }\end{array}$ \\
\hline & $\begin{array}{l}\text { Neutron detection } \\
\text { efficiency }\end{array}$ & $<0.7(\mathrm{EC}+\mathrm{PCAL})$ & n.a. \\
\hline 1 & $\begin{array}{l}\text { Particle ID } \\
\qquad \mathrm{e} / \pi\end{array}$ & Full range & n.a. \\
\hline Central Detector & $\begin{array}{l}\pi / p \\
\pi / K \\
K / p \\
\pi^{0 \rightarrow \gamma \gamma} \\
\eta \rightarrow \gamma \gamma\end{array}$ & $\begin{array}{l}\text { Full range } \\
\text { Full range } \\
<4 \mathrm{GeV} / \mathrm{c} \\
\text { Full range } \\
\text { Full range }\end{array}$ & $\begin{array}{r}<1.25 \mathrm{GeV} / \mathrm{c} \\
<0.65 \mathrm{GeV} / \mathrm{c} \\
<1.0 \mathrm{GeV} / \mathrm{c} \\
\text { n.a. } \\
\text { n.a. }\end{array}$ \\
\hline
\end{tabular}




\section{$12 \mathrm{GeV}$ Schedule}

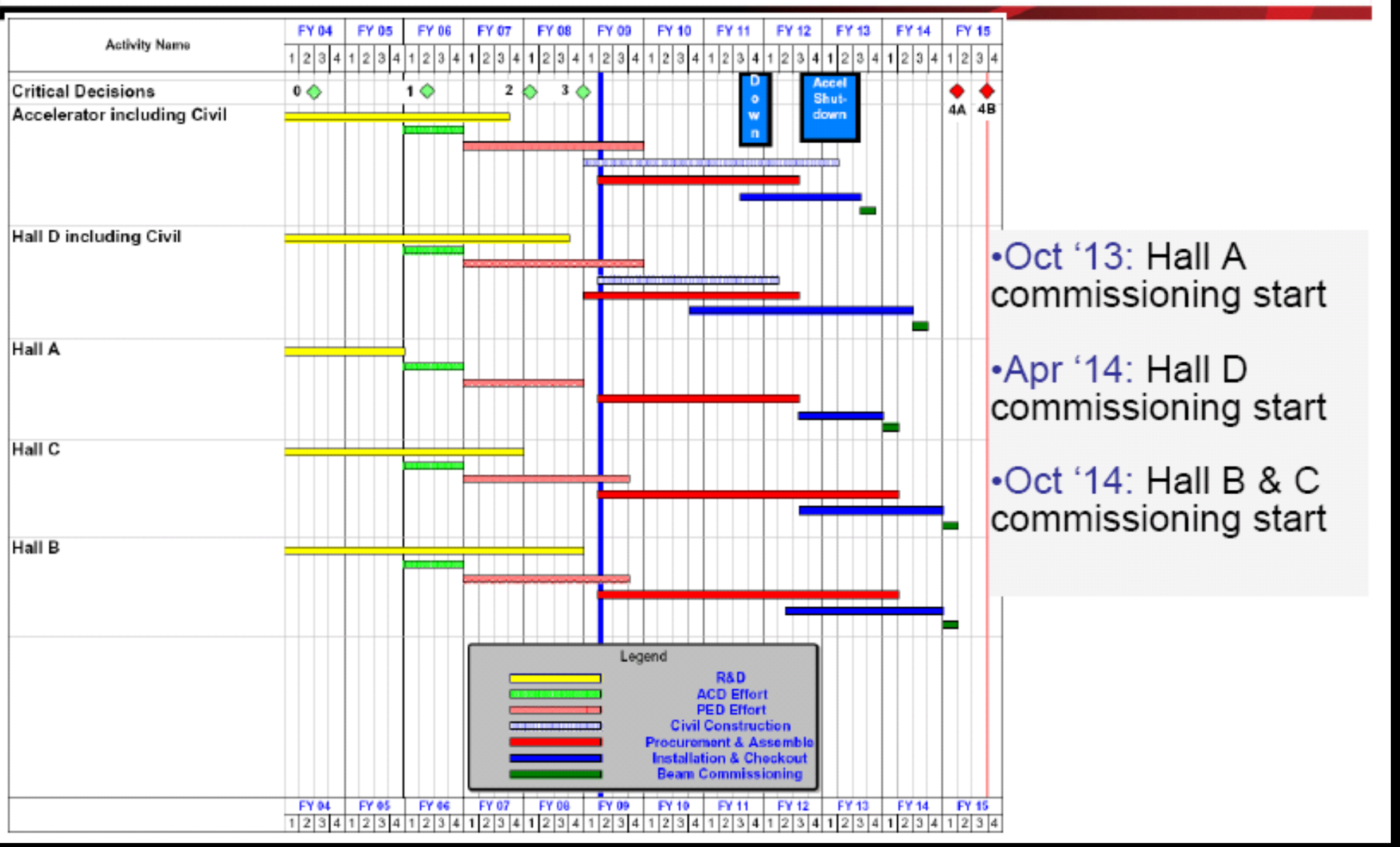

